\title{
Effect of Multiple Visual Feedbacks for Text Entry on Touch screen
}

\author{
Jeeyea Park \\ Dept. of Psychology, Yonsei Univ \\ Yonsei Univ., Seoul, Korea \\ jeeyea86@naver.com
}

\author{
Jiyoung Kwon \\ Dept. of Psychology, Yonsei Univ \\ Yonsei Univ., Seoul, Korea \\ xoxojykwon@gmail.com
}

\author{
Kwang Hee Han \\ Dept. of Psychology, Yonsei Univ. \\ Yonsei Univ., Seoul, Korea \\ khan@yonsei.ac.kr
}

\begin{abstract}
A large amount of study about multisensory feedback for touch screen has been conducted, but multiple unisensory feedback has not. In this study, we investigated both objective and subjective evaluation of multiple visual feedbacks on touch screen. We presented two different visual feedbacks, either alone or combined, for QWERTY text entry. The results showed that the subjective evaluation of visual feedback 1 (Button feedback) was positive, while another visual feedback (Ripples) does not have any effect on evaluations. However, there was significant interaction between the two visual feedbacks. The effect of Button feedback was reduced while Ripples was provided. One possible explanation is that Ripples may not be appropriate for this task. Thus participants might ignore the Ripples feedback. Another is that multiple unisensory feedback might be too complicated, because these visual feedbacks overlap each other and moreover have different information.
\end{abstract}

Touch screen, Visual feedback, Multiple Unisensory feedback, Text Entry

\section{INTRODUCTION}

Direct control of a computer through touch screen reduces cognitive load and overcomes an interface size limitation. However, due to the lack of tactile feedback, we make unnecessary touching and feel uncomfortable during use. Kim found that visual feedback is particularly important for touch screen appliances [1]. Wigdor and colleagues [2] presented Ripples, which enables visualizations around each contact point on a touch screen and shrinks when contact is lifted. They found that user prefer a system utilizing Ripples and Ripples significantly reduced touch errors. We use this visual feedback with Button feedback for text entry on touch screen.

\section{METHOD}

\subsection{Participants}

Twenty-two participants ( 6 females, mean age 22.9 years, ranging from 19 to 29 years) from Yonsei University took part in the experiment.

\subsection{Procedure and Apparatus}

Participants were asked to type the presented phrase using a QWERTY touch keyboard as quickly and as accurately as possible. We measured entry speed using WPM (words-per- minute) for objective evaluation. And, more specific, coordinates of the actual touch were recorded to know touch bias. After the participants completed the tasks under all conditions they were asked to respond to a set of 7-point rating scales for subjective evaluation [3, 4]: Overall user reaction; Perceived usefulness; Perceived ease of use.

The task was programmed in Microsoft Visual Basic 6.0 and presented touch screen (Samsung SyncMaster 743BX). 20 text phrases for the task were taken from MacKenzie and Soukoreff [5].

\subsection{Experimental design}

This study was a $2 \times 2$ factorial, within-subject design. The independent variables were Button feedback and Ripples, each of these variables had two levels: 'present' and 'absent'. Table 1 shows the different variables and levels that comprise each of the four conditions. The conditions were presented in a randomly determined order.

Table 1: Experimental design

\begin{tabular}{|c|c|c|c|}
\hline \multicolumn{3}{|c|}{ Button feedback } \\
\hline \multicolumn{2}{|c|}{ Present } & \multicolumn{2}{c|}{ Absent } \\
\hline \multicolumn{2}{|c|}{ Ripples } & \multicolumn{2}{c|}{ Ripples } \\
\hline Present & Absent & Present & Absent \\
\hline Condition 1 & Condition 2 & Condition 3 & Condition 4 \\
\hline
\end{tabular}

- Feedbacks are lasted for $0.5 \mathrm{~s}$ from released 


\section{RESULTS}

Incomplete phrase data were excluded from the analysis. WPM and subjective rating were evaluated by repeated-measure ANOVAs with the factors Button feedback and Ripples.

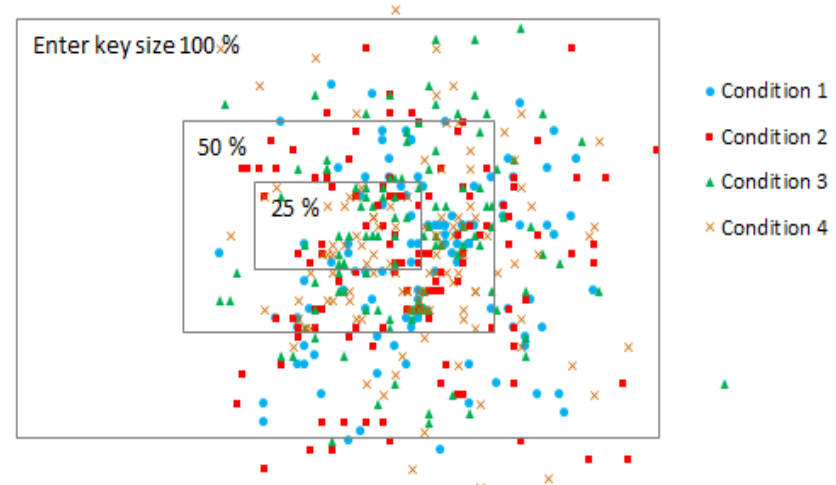

Figure 1: Actual touch location on the Enter key

Table 2: Percentage of right touches on Enter key

\begin{tabular}{|c|c|c|c|}
\hline Enter key & Size $\mathbf{1 0 0} \%$ & Size $\mathbf{5 0} \%$ & Size $\mathbf{2 5} \%$ \\
\hline Condition 1 & $98 \%$ & $62 \%$ & $15 \%$ \\
\hline Condition 2 & $94 \%$ & $58 \%$ & $19 \%$ \\
\hline Condition 3 & $98 \%$ & $69 \%$ & $24 \%$ \\
\hline Condition 4 & $96 \%$ & $68 \%$ & $23 \%$ \\
\hline
\end{tabular}

In the objective evaluation, using WPM, there was no main effect of visual feedback and interaction $[F s<1]$. This is not the same as results from previous research on Ripples [2]. But the actual touch location on Enter key (fig. 1) show us some touch bias. Touch bias means represent the distance from the center of the key to actual touch location. More bias was observed when Button feedback present conditions than absent (Table. 2). This means Button feedback was obvious so makes people more comfortable.

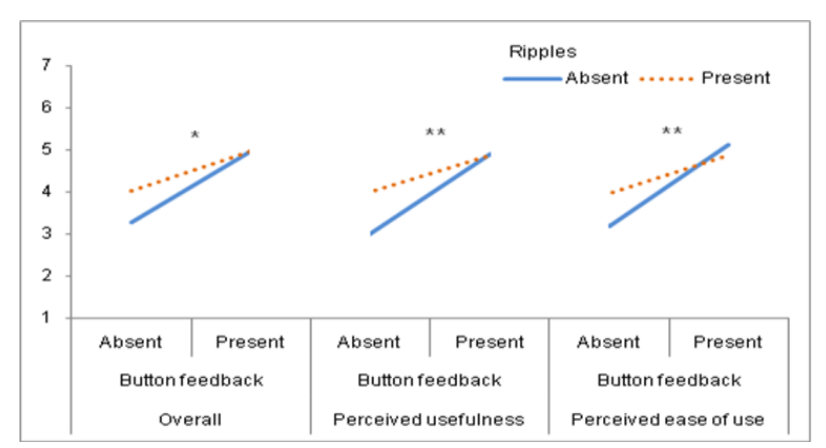

Figure 2: Button feedback $x$ Ripples interaction for Subjective Evaluation

In the subjective evaluation, the ANOVA on all of the questionnaires showed positive responses for Button feedback present condition than absent condition [overall user reaction, $F(1,21)=65.48, p$
$<.001, \eta_{p}^{2}=.76$; perceived usefulness, $F(1,21)=$ 84.84, $p<.001, \eta_{p}^{2}=.80$; perceived ease of use, $\left.F(1,21)=52.20, p<.001, \eta_{p}^{2}=.71\right]$ But this response was not shown with Ripples $(p>.05)$.

The Button feedback $x$ Ripples interaction was significant [overall user reaction, $F(1,21)=5.80, p$ $<.05, \eta_{p}^{2}=.22$; perceived usefulness, $F(1,21)=$ $9.91, p<.01, \eta_{p}^{2}=.32$; perceived ease of use, $F(1$, $21)=13.66, p<.01, \eta_{p}{ }^{2}=.39$ ] (fig. 2). The rating of multiple visual feedbacks (condition 4) was similar to condition 3 (only Button feedback presented), and the effect of Button feedback was reduced while Ripples was provided.

\section{DISCUSSION}

The results of the study revealed effect of multiple visual feedbacks on touch screen. There were no differences in WPM, but, subjective evaluation showed significant differences based on feedback conditions. The effect of Button feedback was positive and there was an interaction between Button feedback and Ripples. The two feedbacks had different information. Thus, the user might ignore less attractive feedback (i.e. Ripples) instead of integrating them. Also actual touch location was not different under the Ripples condition levels, but under the level of Button feedback. Possible cause of no significant effect of Ripples is that this feedback might not be appropriate to the task. Ripples gave users the reason for a 'miss' targeting by showing an exact contacted position. But in this study, the error rate for a complete phrase was very low. Moreover the size of Ripples was bigger than key spacing, possibly overcomplicating the task for some. We assume that smaller sized Ripples is useful in more difficult task.

\section{REFERENCES}

[1] Kim, J.H. (2009) Multimodal Feedback Usability Analysis on Touchscreen-Applied Mobile Device. Master. Hongik University

[2] Wigdor, D. Williams, S. Cronin, M. Levy, R. White, K. Mazeev, M. and Benko, H. (2009) Ripples: Utilizing Per-Contact Visualizations to Improve User Interaction with Touch Displays. In Proceedings of UIST 09, Victoria, British Columbia, 4-7 October, Canada.

[3] Questionnaire for User interaction Satisfaction. http://www.lap.umd.edu/quis/index.html.

[4] Davis, F.D. (1989) Perceived Usefulness, Perceived Ease of Use, and User Acceptance of Information Technology. MIS Quarterly, 13(3), 319-340.

[5] MacKenzie, S. Soukoreff, R. Phrase Sets for Evaluating Text Entry Techniques. In Proceedings of $\mathrm{CHI}$ 03, Ft. Lauderdale, 5-10 April, Florida, USA. 